\title{
Lattice QCD study of $g_{A}^{N^{*} N^{*}}$ with two flavors of dynamical quarks
}

\author{
T. T. Takahashi and T. Kunihiro \\ Yukawa Institute for Theoretical Physics \\ Kyoto University \\ Kitashirakawa-Oiwakecho, Sakyo, Kyoto 606-8502, Japan
}

\begin{abstract}
We report the first lattice QCD result of the axial charge of $\mathrm{N}(1535)$, $g_{A}^{N^{*} N^{*}}$. The measurement is performed with two flavors of dynamical quarks employing the renormalization-group improved gauge action at $\beta=1.95$ and the mean-field improved clover quark action with the hopping parameters, $\kappa=0.1375,0.1390$ and 0.1400 . In order to avoid the signal contaminations by $\mathrm{N}(1650)$ lying just $100 \mathrm{MeV}$ above $\mathrm{N}(1535)$, we construct $2 \times 2$ correlation matrices and diagonalize them so that clear signal separation can be found. The wraparound contributions in the correlator, which can be another source of signal contamination, are eliminated by imposing the Dirichlet boundary condition in the temporal direction. We find that the axial charge of $\mathrm{N}(1535)$ takes small values as $g_{A}^{N^{*} N^{*}} \sim 0.2$, independent of quark masses, in the pionmass range of 0.7 to $1.1 \mathrm{GeV}$.
\end{abstract}

\section{Introduction}

Chiral symmetry is an approximate global symmetry in Quantum ChromoDynamics (QCD), and the symmetry and its spontaneous breaking is one of the key ingredients in the low-energy hadron physics. For instance, all the hadrons can be classified into representations of $S U\left(N_{f}\right)_{L} \times S U\left(N_{f}\right)_{R}$. Once we fix the representations, it gives strong constraints to low-energy effective lagrangians and possible terms are uniquely determined besides overall constants. To embody chiral symmetry in effective lagrangians, we have two famous ways; the linear and the non-linear representations. The non-linear representation has been well studied and successful especially in the context of the chiral perturbation theory. The linear representation with scalar mesons as chiral partners of Nambu-Goldstone bosons would be important around the chiral restoration point at high temperature/density. 
As for the realization of chiral representations in the baryon sector in the linear representation, there could be naively two ways $[1,2]$. One is the naive assignment and the other is the so-called mirror assignment introduced by DeTar and Kunihiro [1]. We can find several important differences between these two assignments in the couplings or in the nucleon masses. For example, the nucleon and its parity partner belong to the same chiral multiplet and there can exist chirally-invariant mass terms of nucleons in the mirror assignment [1]. Due to the mass terms, nucleons can be massive even when the chiral condensate takes a small value or zero, whereas nucleon masses are simply proportional to the chiral condensate in the naive assignment $[1,2]$, which would be the most important difference between the naive and mirror cases. Such differences play crucial roles at finite temperature/density systems and it should be revealed directly from QCD. In order to clarify which assignment is natural, it would be advantageous to measure the axial charge of $\mathrm{N}(1535)$, which we assume as the chiral partner of N(940), because the axial charges of $\mathrm{N}(940)$ and $\mathrm{N}(1535)$ are sensitive to the chiral structure of baryons $[1,2]$ and have the same (different) signs in the naive (mirror) assignments.

In this report, we show the first unquenched lattice QCD study of $g_{A}^{N^{*} N^{*}}$ as well as $g_{A}^{N N}$. (For the details, see [3].) We employ $16^{3} \times 32$ lattice with two flavors of dynamical quarks, generated [4] with the renormalization-group improved gauge action at $\beta=1.95$ and the mean field improved clover quark action with the clover coefficient $c_{\mathrm{SW}}=1.530$. The calculations are done with the hopping parameters, $\kappa_{\text {sea }}, \kappa_{\text {val }}=0.1375,0.1390$ and 0.1400 .

\section{Lattice QCD formulations and results}

$\mathrm{N}(1535)$ is the ground-state nucleon in $\frac{1}{2}^{-}$channel. Though a ground state signal can be in principle isolated using a large Euclidean time separation between the source and the sink points in correlators, we could suffer from the signal contamination by $\mathrm{N}(1650)$ lying just $100 \mathrm{MeV}$ above. With the aim to separate the signals in a proper way and to optimize operators, we diagonalize correlation matrices constructed with two independent operators; $N_{1}(x) \equiv$ $\varepsilon_{\mathrm{abc}} u^{a}(x)\left(u^{b}(x) C \gamma_{5} d^{c}(x)\right), N_{2}(x) \equiv \varepsilon_{\mathrm{abc}} \gamma_{5} u^{a}(x)\left(u^{b}(x) C d^{c}(x)\right)$. Here, $u(x)$ and $d(x)$ are the Dirac spinors for $\mathrm{u}$ - and d- quarks, respectively, and a,b,c denote the color indices. We eliminate wraparound effects, which could be another possible sources of contamination, imposing the Dirichlet boundary condition in the temporal direction.

With the optimized operators $\mathcal{N}(x)$, we can obtain vector(axial) charges 
$g_{V}\left(g_{A}\right)$ as follows.

$$
g_{V} \rightarrow \frac{\operatorname{tr} \gamma_{4} \Gamma\left\langle\mathcal{N}\left(t_{\mathrm{snk}}\right) V_{4}(t) \overline{\mathcal{N}}\left(t_{\mathrm{src}}\right)\right\rangle}{\operatorname{tr} \Gamma\left\langle\mathcal{N}\left(t_{\mathrm{snk}}\right) \overline{\mathcal{N}}\left(t_{\mathrm{src}}\right)\right\rangle} \quad\left(t_{\mathrm{snk}} \gg t \gg t_{\mathrm{src}}\right)
$$

and

$$
g_{A} \rightarrow \frac{\operatorname{tr} \gamma_{5} \gamma_{3} \Gamma\left\langle\mathcal{N}\left(t_{\mathrm{snk}}\right) A_{3}(t) \overline{\mathcal{N}}\left(t_{\mathrm{src}}\right)\right\rangle}{\operatorname{tr} \Gamma\left\langle\mathcal{N}\left(t_{\mathrm{snk}}\right) \overline{\mathcal{N}}\left(t_{\mathrm{src}}\right)\right\rangle} \quad\left(t_{\mathrm{snk}} \gg t \gg t_{\mathrm{src}}\right),
$$

with $\Gamma \equiv \frac{1+\gamma_{4}}{2}$. Here, $A_{\mu}(t) \equiv \sum_{\mathbf{x}} \bar{u}(x) \gamma_{\mu} \gamma_{5} u(x)-\bar{d}(x) \gamma_{\mu} \gamma_{5} d(x)$ and $V_{\mu}(t) \equiv$ $\sum_{\mathbf{x}} \bar{u}(x) \gamma_{\mu} u(x)-\bar{d}(x) \gamma_{\mu} d(x)$ are the zero-momentum projected axial and vector currents, and the traces are taken over spinor indices. All the unwanted quantities, such as the normalization factors, are all canceled out between the denominator and the numerator.
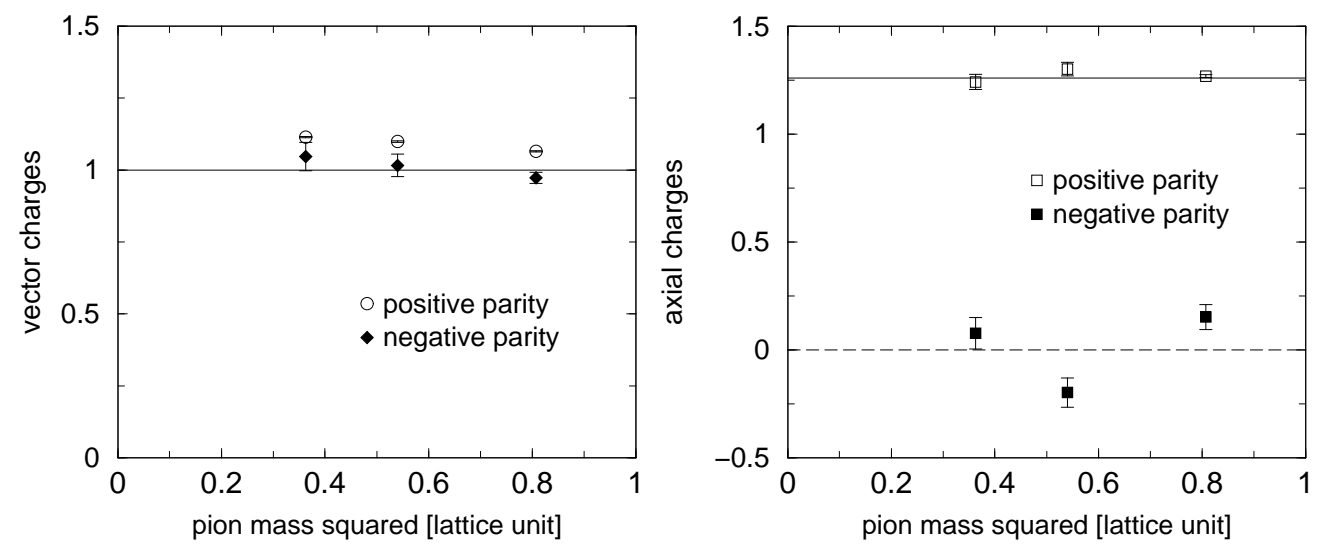

Figure 1: The renormalized vector and axial charges of the positive- and the negative-parity nucleons are plotted as the function of the squared pion mass $m_{\pi}^{2}$. The left panel shows the results of the vector charges and the right panel the results of the axial charges. In the left panel, the solid line is drawn at $g_{V}=1$ for reference. In the right panel, the solid line is drawn at $g_{A}=1.26$ and the dashed line is drawn at $g_{A}=0$.

The renormalization factors for bilinear operators are determined with the constants listed in Ref. [4]. We plot in the left panel in Fig. 1 the vector charges of the positive- and the negative-parity nucleons, which should be unity. The open (filled) symbols denote the vector charges of the positive(negative-) parity nucleon at each hopping parameter. We can find about $10 \%$ deviations from unity, which can be considered to come from the systematic errors in the renormalization factors. We should then take into account at least $10 \%$ systematic errors in our results. The axial charges of 
the positive-parity nucleon at each hopping parameter are plotted in the right panel. They are shown as the open symbols. One can find the good agreement between the lattice data and 1.26, the experimental value.

We finally show the axial charges of the negative-parity nucleon in the right panel. One finds at a glance that they take quite small values, as $g_{A}^{N^{*} N^{*}} \sim 0.2$ and that even the sign is quark-mass dependent. While the wavy behavior might come from the sensitiveness of $g_{A}^{N^{*} N^{*}}$ to quark masses, this behavior may indicate that $g_{A}^{N^{*} N^{*}}$ is rather consistent with zero. The small $g_{A}^{N^{*} N^{*}}$ reflects the interesting chiral structure of baryons $[1,2,5-7]$.

The present quark masses are unfortunately so heavy that their related pion masses are $700 \mathrm{MeV} \sim 1.1 \mathrm{GeV}$. In order to reveal the chiral structure, much lighter $\mathrm{u}, \mathrm{d}$ quarks are indispensable. The study of the axial charge of Roper or N(1650) as well as the inclusion of strange sea quarks could also cast light on the low-energy chiral structure of baryons. They are left for further study.

\section{Acknowledgments}

All the numerical calculations were performed with NEC SX-8 at CMC, Osaka university and at YITP, Kyoto university. The unquenched gauge configurations employed in our analysis were all generated by CP-PACS collaboration [4].

\section{References}

[1] C. DeTar and T. Kunihiro, Phys. Rev. D 39, 2805 (1989).

[2] D. Jido et al. Nucl. Phys. A 671, 471 (2000)

[3] T. T. Takahashi and T. Kunihiro, in preparation.

[4] A. Ali Khan et al. [CP-PACS Collaboration], Phys. Rev. D 65, 054505 (2002) [Erratum-ibid. D 67, 059901 (2003)]

[5] D. Jido, T. Hatsuda and T. Kunihiro, Phys. Rev. Lett. 84, 3252 (2000)

[6] R. L. Jaffe, D. Pirjol and A. Scardicchio, Phys. Rept. 435, 157 (2006)

[7] L. Y. Glozman, Phys. Rept. 444, 1 (2007) 